\title{
CYLINDRICAL PROBABILITIES AND THE DIFFERENTIATION OF VECTOR MEASURES
}

\author{
BRIAN JEFFERIES
}

\section{§1. Introduction}

There are many results in probability theory on vector spaces which rely implicitly on the approximation of a given cylindrical probability by cylindrical probabilities with moments; for example, this is the basic idea behind the proof of the Radon equivalence of the weak and strong topologies of a metrizable space (Schwartz [13] p. 162). The technique of approximation by cylindrical measures with moments can be systematically developed. In particular, it follows that if each member of a family of cylindrical probabilities with moments is decomposable, then the limits of these cylindrical probabilities are often regular and so they are $\sigma$-additive.

An application of this method is related to the work of $\mathrm{L}$. Schwartz [11], [12] dealing with the notion of " $p$-radonifying" maps. The well-known theorem due to Sazanov (see [13]) asserts that a continuous linear operator between Hilbert spaces is Hilbert-Schmidt if and only if it maps every cylindrical probability scalarly concentrated on bounded sets into a Radon measure (i.e. it is "o-radonifying"). However, there are maps between Banach spaces which are even nuclear but not o-radonifying. In fact, the maps need only be a little better than nuclear maps to be o-radonifying. Furthermore, there are absolutely summing maps between Banach space which are not 1-radonifying. This phenomenon is related to the RadonNikodým property of the range space [12].

Since nuclear and absolutely summing maps send vector measures into indefinite integrals (i.e. they are "Nikodýmising"), the approximation argument mentioned above allows us to find classes of cylindrical probabilities for which nuclear and absolutely summing maps are regularizing. The method has the advantage of relying only on the notions of summability and boundedness in a locally convex space, so there is no need to

Received January 5, 1987. 
use norms or quasinorms as in [11].

Now if the space $E$ has the Radon-Nikodým property and the cylindrical probabitity $\mu$ is the limit of certain cylindrical probabilities arising from vector measures, then $\mu$ is regular and so $\sigma$-additive. Furthermore, any sufficiently regular cylindrical probability arises in this fashion. The internal structure of a conuclear space is determined by a collection of nuclear maps defined in it, so applying the argument above for nuclear maps allows us to give a necessary and sufficient condition for which a cylindrical probability is regular, and so $\sigma$-additive on a conuclear space. This condition does not involve the continuity of the linear random function associated with $\mu$ as in the Minlos theorem. It is feasible that when the approximating family is readily available, the condition will be easier to apply than Minlos's result.

\section{§2. Preliminaries}

In this section some terminology and notation are fixed, and results concerning cylindrical probabilities are reviewed.

The conventions of Schaefer [10] for locally convex spaces are followed generally. A family $\mathscr{B}$ of subsets of a locally convex space $E$ is called a convex bornology if

(a) the union of any two sets in $\mathscr{B}$ also belongs to $\mathscr{B}$;

(b) any subset of a set in $\mathscr{B}$ also belongs to $\mathscr{B}$;

(c) the set $\cup \mathscr{B}$ is dense in $E$;

(d) the closed, balanced convex hull $\overline{\mathrm{bco}}(B)$ of a set $B \in \mathscr{B}$ also belongs to $\mathscr{B}$;

(e) if $a>0, B \in \mathscr{B}$, then $a B \in \mathscr{B}$.

If condition (c) is not satisfied, $\mathscr{B}$ is said to be a convex bornology in $E$. A base for the convex bornology $\mathscr{B}$ is a subfamily $\mathscr{A}$ of $\mathscr{B}$ such that every element of $\mathscr{B}$ is contained in an element of $\mathscr{A}$. The convex bornology $\mathscr{B}$ is said to be complete if it has a base consisting of disked (i.e. balanced and convex) sets $B \in \mathscr{B}$ for which $E_{B}$ (the normed space $\left.\left(\cup_{n \in N} n B, p_{B}\right)\right)$ is complete. The standard convex bornologies of a locally convex space $E$ include the family $\mathscr{B}_{0}$ of bounded subsets of $E$, the family $\mathscr{C} \mathscr{C}$ of subsets of compact, convex subsets of $E$, and the family $\mathscr{W} \mathscr{C} \mathscr{C}$ of subsets of weakly compact, convex subsets of $E$. If $E$ is quasi-complete, then $\mathscr{C} \mathscr{C}=\mathscr{C}(\mathscr{W} \mathscr{C} \mathscr{C}$ $=\mathscr{W} \mathscr{C}$ ) the family of subsets of compact (weakly compact) subsets of $E$.

Given a complete convex bornology $\mathscr{B}$ in $E$, we also consider two 
other convex bornologies generated by $\mathscr{B}$. A set $A \subset E$ is said to be

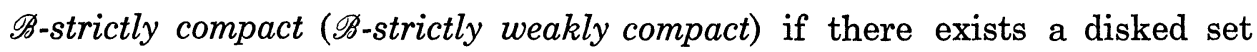
$B \in \mathscr{B}$ such that $A \subset B$ and $A$ is compact (weakly compact) in the normed

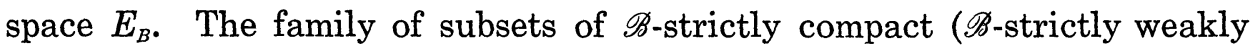
compact) sets is denoted by $\mathscr{B} \mathscr{C}(\mathscr{B} \mathscr{W} \mathscr{C})$. These families are convex bornologies, because $\mathscr{B}$ is assumed complete.

The following two results are proved in [6].

THEOREM 2.1. Let $\mathscr{B}$ be a complete convex bornology on the locally convex space $E$. Let $u: E^{\prime} \rightarrow F$ be a linear map into the locally convex space $F$.

Suppose that $u$ is continuous for the topology of uniform convergence on $\mathscr{B}$, and for some $B \in \mathscr{B}$, the restriction of $u$ to $B^{\circ}$ (the polar of $B$ in $E^{\prime}$ ) is relatively $\tau\left(E^{\prime}, E\right)$-continuous.

Then $u$ is $\tau\left(E^{\prime}, E\right)$-continuous into $F$.

THEOREM 2.2. Let $\mathscr{B}$ be a complete convex bornology on the locally convex space $E$. Let $u: E_{\tau}^{\prime} \rightarrow F$ be a continuous linear map into the Banach space $F$ which is also continuous for the topology of uniform convergence on $\mathscr{B}$.

Then $u$ is continuous for the topology of uniform convergence on $\mathscr{B} \mathscr{W} \mathscr{C}$ if and only if there exists $B \in \mathscr{B}$ such that $u\left(B^{\circ}\right)$ is relatively weakly compact in $F$.

Theorem 2.1 may be viewed as Grothendieck's completeness theorem [10] IV, 6.2 for the bornological setting. Here only the local completeness of the bornology $\mathscr{B}$ is required, instead of the completeness of the whole space $E$.

The generality of locally convex spaces and bornologies considered here is required for examples which arise naturally in applications. For example, let $\mathscr{L}_{s}(E, F)$ denote the space of continuous linear operators between a Fréchet space $E$ and a Banach space $F$, equipped with the strong operator topology. Then $\mathscr{L}_{s}(E, F)$ is not complete or metrizable, and it has no natural norm structure. However, the convex bornology generated by the sets of the form $\{u: \sup \{|u(x)|: x \in B\} \leqq 1\}$ for $B$ bounded in $E$ is complete, and it immediately suggests itself as a suitable object of study for probability theory on $\mathscr{L}_{s}(E, F)$.

If $\mathscr{B}$ is a convex bornology on $E$, then the topology on $E^{\prime}$ of uniform convergence on sets in $\mathscr{B}$ is locally convex. The space $E^{\prime}$ with this topology 
is denoted by $E_{\mathscr{B}}^{\prime}$.

Let $\mathscr{Z}(E)$ denote the algebra of cylinder sets of the locally convex space $E$ (sets of the form $\phi^{-1}(B)$ for $\phi: E \rightarrow R^{k}$ continuous and linear, and $B$ a Borel subset of $R^{k}$ ) and $\mathscr{C} \mathscr{L}(E)$ the $\sigma$-algebra generated by $\mathscr{Z}(E)$. The Borel $\sigma$-algebra of a Hausdorff topological space $T$ is denoted by $\mathscr{B O}(T)$ (the smallest $\sigma$-algebra containing the open sets).

A cylindrical probability on $E$ is an additive set function $\mu: \mathscr{Z}(E) \rightarrow$ $[0,1]$ such that for each continuous linear map $\phi: E \rightarrow R^{k}, k \in N$, the set function $\mu \circ \phi^{-1}: \mathscr{B} \mathcal{O}\left(\boldsymbol{R}^{k}\right) \rightarrow[0,1]$ defined by $\mu \circ \phi^{-1}(A)=\mu\left(\phi^{-1}(A)\right), A \in \mathscr{B} \mathcal{O}\left(\boldsymbol{R}^{k}\right)$ is a $\sigma$-additive probability measure.

The algebraic dual $E^{\prime *}$ of $E^{\prime}$ is isomorphic to the completion of $E$ in $\sigma\left(E, E^{\prime}\right)$. Given a cylindrical probability $\mu: \mathscr{Z}(E) \rightarrow[0,1]$, there exists a unique $\sigma$-additive probability $\mu_{\sigma}: \mathscr{C} \mathscr{L}\left(E^{\prime *}\right) \rightarrow[0,1]$ such that $\mu_{\sigma}(A)=$ $\mu(A \cap E)$ for every $A \in \mathscr{Z}\left(E^{\prime *}\right)$ (identifying $E$ with its canonical image in $\left.E^{* *}\right)$.

Let $0<p<\infty$. A cylindrical probability $\mu$ on $E$ has (weak) $p$-th order moments if for all $\xi \in E^{\prime}, \mu\left(|\xi|^{p}\right)<\infty$ (since $x \rightarrow|\langle x, \xi\rangle|^{p}, x \in E$ is $\mathscr{Z}(E)$-measurable, the integral is well-defined). Now let $\rho$ be a locally convex topology on $E^{\prime}$ and $\mathscr{B}$ a bornology on $E$. We say that $\mu$ has $(\rho, p)$-moments $((\mathscr{B}, p)$-moments $)$ if $\mu$ has $p$-th order moments and the map $\xi \rightarrow \mu\left(|\xi|^{p}\right), \xi \in E^{\prime}$ is continuous on $E_{\rho}^{\prime}\left(E_{\circledast}^{\prime}\right)$.

The cylindrical probabilities with $(\tau, 1)$-moments, (here $\tau$ is the Mackey topology $\tau\left(E^{\prime}, E\right)$ on $\left.E^{\prime}\right)$ are of special interest. Suppose that $\mu$ has $(\tau, 1)$ moments, and denote the identity map on $E^{\prime *}$ by $\iota_{\sigma}$. Then $\iota_{\sigma}$ is $\mu_{\sigma}$-integrable on $E^{\prime *}$, and the values of $\iota_{\sigma} \mu_{\sigma}$ on $\mathscr{C} \mathscr{L}\left(E^{* *}\right)$ are all contained in a weakly compact convex subset of $E$. Furthermore, if $\phi: E \rightarrow R^{k}$ is a continuous linear map, $k \in N$, then on $\mathscr{B} \mathcal{O}\left(\boldsymbol{R}^{k}\right)$,

$$
\mu \circ \phi^{-1}=\mu_{\sigma} \circ\left(d \phi \circ \iota_{\sigma} \mu_{\sigma} / d \mu_{\sigma}\right)^{-1} \text {. }
$$

In this sense, $\mu$ is the "distribution" of a density for the vector measure $\iota_{\sigma} \mu_{\sigma}$ with respect to $\mu_{\sigma}$ ( $\mu$ is said to be the $\left(\iota_{\sigma} \mu_{\sigma}, \mu_{\sigma}\right)$-distribution). For more details and references, see [7].

A cylindrical probability $\mu$ on the locally convex space $E$ is said to be scalarly concentrated on the family $\mathscr{F}$ of subsets of $E$, if for every $\varepsilon>0$, there exists a set $F \in \mathscr{F}$ such that $\left(\mu \circ \xi^{-1}\right)^{*}(\xi(F)) \geqq 1-\varepsilon$, for all $\xi \in E^{\prime}$. The cylindrical probability $\mu$ is cylindrically concentrated on $\mathscr{F}$ if for every $\varepsilon>0$, there exists a set $F \in \mathscr{F}$ such that $\left(\mu \circ \phi^{-1}\right) *(\phi(F)) \geqq 1-\varepsilon$, 
for all continuous linear $\phi: E \rightarrow R^{k}, k \in N$.

The following result appears in [15].

Theorem 2.3. Let $E$ be a complete locally convex space and let $p>1$. If the cylindrical probability $\mu$ has $p$-th order moments, and if $\mu$ is scalarly concentrated on weakly compact sets, then $\mu$ has $(\tau, p)$-moments.

In particular $\mu$ has $(\tau, 1)$-moments.

Theorems 2.1 and 2.2, and an argument analogous to the proof of the above theorem can be applied to yield the corresponding result for bornologies (strengthening a similar statement in [7]).

THEOREM 2.4. Let $\mathscr{B}$ be a complete convex bornology on the locally convex space $E$, and let $p>1$. If the cylindrical probability $\mu$ has $(\mathscr{B}, p)$ moments, and if $\mu$ is scalarly concentrated on weak compact convex subsets of $E$, then $\mu$ has $(\mathscr{B} \mathscr{W} \mathscr{C}, p)$-moments.

Proof. Let $M: E^{\prime} \rightarrow L^{\circ}\left(\mu_{\sigma}\right)$ be the linear random function associated with $\mu$ [13]. Since $\mu$ has $p$-th order moments, $M\left(E^{\prime}\right) \subset L^{p}\left(\mu_{\sigma}\right)$ and $\mu$ has $(\mathscr{B} \mathscr{W} \mathscr{C}, p)$-moments if and only if $M: E_{\mathscr{G} \mathscr{W}}^{\prime} \rightarrow L^{p}\left(\mu_{\sigma}\right)$ is continuous.

Now $\mu$ has $(\mathscr{B}, p)$-moments, so $M: E_{\mathscr{B}}^{\prime} \rightarrow L^{p}$ is continuous. The unit ball of $L^{p}$ is weakly compact for $p>1$, so to prove $M: E_{\mathscr{Q} W \mathscr{B}}^{\prime} \rightarrow L^{p}$ is continuous, it suffices to prove $M: E_{\tau}^{\prime} \rightarrow L^{p}$ is continuous, by Theorem 2.2. By Theorem 2.1 and [10] p. 158, this will be true when for some $B \in \mathscr{B}$, the restriction of $M$ to $B^{\circ}$ is continuous in the relative Mackey topology $\tau\left(E^{\prime}, E\right)$, and for the weak topology $\sigma\left(L^{p},\left(L^{p}\right)^{\prime}\right)$ of $L^{p}$. We now establish this condition.

Since $M: E_{\mathscr{B}}^{\prime} \rightarrow L^{p}$ is continuous, a disked set $B \in \mathscr{B}$ can be chosen so that $M\left(B^{\circ}\right)$ is contained in the unit ball of $L^{p}$. Let $p^{\prime}=p /(p-1)$, and let $g \in L^{p^{\prime}}$. Define a linear functional $u_{g}: E^{\prime} \rightarrow R$ by

$$
u_{g}(\xi)=\mu_{\sigma}(M(\xi) g), \quad \xi \in E^{\prime} .
$$

We show that $u_{g} \mid B^{\circ}$ is continuous in the relative Mackey topology on $B^{\circ}$.

Given $\varepsilon>0$, choose $n>0$ such that

$$
|g|^{p^{\prime}} \mu_{\sigma}(\{|g|>n\})<(\varepsilon / 3)^{p^{\prime}} .
$$

Then for each $\xi \in B^{\circ}$,

$$
\left|u_{g}(\xi)\right| \leqq\left|M(\xi) g \mu_{o}\{|g| \leqq n\}\right|+\varepsilon / 3,
$$


because $M\left(B^{\circ}\right)$ is contained in the unit ball of $L^{p}\left(\mu_{\sigma}\right)$. Now $\mu$ is scalarly concentrated on weakly compact convex subsets of $E$, so for some disked $K \in \mathscr{W} \mathscr{C} \mathscr{C}$

$$
\mu \circ \xi^{-1}(\xi(K)) \geqq 1-(\varepsilon / 3 n)^{p^{\prime}}, \quad \xi \in E .
$$

On the other hand, if $\xi_{\sigma}$ denotes the extension of $\xi$ to $E^{\prime *}$, then

$$
\begin{aligned}
\mu_{\sigma}(|M(\xi)|) & =|M(\xi)| \mu_{\sigma}\left(\xi_{\sigma}^{-1}(\xi(K))\right)+|M(\xi)| \mu_{\sigma}\left(\xi_{\sigma}^{-1}[\xi(K)]^{c}\right) \\
& \leqq p_{K}(\xi)+\|M(\xi)\|_{p}\left[\mu_{\sigma}\left(\xi_{\sigma}^{-1}[\xi(K)]^{c}\right)\right]^{1 / p^{\prime}} .
\end{aligned}
$$

Consequently, if $p_{K}(\xi)<\varepsilon / 3 n, \xi \in B^{\circ}$, then $\left|u_{g}(\xi)\right|<\varepsilon$; the theorem is proved.

If $p \geqq 1, E_{\mathscr{B}}^{\prime}$ is barrelled and if $\mu$ is scalarly concentrated on $\mathscr{B}$ and has $p$-th order moments then $\mu$ has $(\mathscr{B}, p)$-moments. The requirement that $E_{\mathscr{B}}^{\prime}$ is barrelled cannot be relaxed [7]. These results give an effective method for showing that a cylindrical probability with moments has continuous moments.

Two main topologies will be considered on the space $\mathrm{P}(E)$ of cylindrical probabilities on the locally convex space $E$. A bounded continuous cylinder function $f: E \rightarrow R$, is a function of the form $f=g \circ \phi$, where $g$ : $\boldsymbol{R}^{k} \rightarrow \boldsymbol{R}$ is bounded and continuous, and $\phi: E \rightarrow \boldsymbol{R}^{k}, k \in N$ is continuous and linear. If $\mu \in \mathrm{P}(E)$, then $\mu(f)$ is uniquely defined by $\mu(f)=\mu \circ \phi^{-1}(g)$. Denote by $\mathrm{C}_{c}(E)$ the collection of bounded continuous cylinder functions on $E$. The cylindrical topology $\gamma$ on $\mathrm{P}(E)$ is the topology of pointwise convergence on $\mathrm{C}_{c}(E)$; that is, the coarsest topology for which the map $\mu \rightarrow \mu(f), \mu \in \mathrm{P}(E)$ is continuous for each $f \in \mathrm{C}_{c}(E)$. A sequence $\left(\mu_{n}\right)_{n \in N} \subset$ $\mathrm{P}(E)$ converges to $\mu \in \mathrm{P}(E)$ in the topology $\gamma$ if and only if the Fourier transform $\hat{\mu}_{n}$ of $\mu_{n}, n \in N$ converge pointwise on $E^{\prime}$ to the Fourier transform $\hat{\mu}$ of $\mu$.

A finer topology $\sigma_{c}$ is specified by saying that a net $\left(\mu_{i}\right)_{i \in I}$ converges to $\mu$ in $\left(\mathrm{P}(E), \sigma_{c}\right)$ if and only if $\left(\mu_{i}\right)_{\sigma}(C) \rightarrow \mu_{\sigma}(C)$ for every $C \in \mathscr{C} \mathscr{L}\left(E^{\prime *}\right)$. Both of these topologies are coarser than the topology of convergence in variation on $\mathrm{P}(E)$.

Let $T$ be a Hausdorff topological space and let $\mathscr{F}$ be a collection of subsets of $T$. A Borel probability $\nu: \mathscr{B O}(T) \rightarrow[0,1]$ is said to be $\mathscr{F}$-regular if

i) $\nu(A)=\sup \{\nu(C): C \subseteq A, C$ is closed in $T\}$ for each $A \in \mathscr{B} O(T)$, and 
(ii) $\sup \left\{\nu^{*}(F): F \in \mathscr{F}\right\}=1$.

Let $\rho$ be a topology on the locally convex space $E$ which is finer than $\sigma\left(E, E^{\prime}\right)$. Then Prokhorov's theorem asserts that a cylindrical probability is cylindrically concentrated on the family $\mathscr{F}$ of $\rho$-compact sets, if and only if it has a unique extension to an $\mathscr{F}$-regular Borel measure on $E_{\rho}$. Indeed, it is usually stated for $\rho=\sigma\left(E, E^{\prime}\right)$, but the topologies of $\sigma\left(E, E^{\prime}\right)$ and $\rho$ coincide on $\rho$-compact sets, so the unique extension exists [13].

\section{§3. Limits of cylindrical probabilities}

The two topologies $\sigma_{c}$ and $\gamma$ are used in this section to relate the cylindrical concentration of a family of cylindrical probabilities to the cylindrical concentration of elements in its closure. The main aim of this technique is to formulate conditions showing when a given cylindrical probability is $\sigma$-additive.

In this respect, the cylindrical topology $\gamma$ is rather weak, so that it must be supplemented by additional restrictions on the size of the approximating family of cylindrical probabilities.

The next lemma shows that a cylindrical probability is regular when it is concentrated on a sufficiently small subspace of a locally convex space. Its proof follows directly from the general theory of Souslin spaces [13]. Alternatively, the Banach-Dieudonné theorem can be used in the present setting to give a more elementary proof.

LEMMA 3.1. Let $\mathscr{B}$ be a complete convex bornology in the locally convex space $E$. The following conditions are equivalent for a cylindrical probability $\mu$ on $E$.

(i) $\mu$ is cylindrically concentrated on $\mathscr{B} \mathscr{C}$.

(ii) There exists a family $\left(B_{n}\right)_{n \in N} \subset \mathscr{B}$ of closed disked sets such that $E_{B_{n}}$ is separable for each $n \in N$, and $\mu_{\sigma}^{*}\left(\cup_{n \in N} E_{B_{n}}\right)=1$.

(iii) $\mu$ extends to a unique $\mathscr{B} \mathscr{C}$-regular Borel measure on $E_{\beta}$.

The condition that $\mu$ is cylindrically concentrated on $\mathscr{B} \mathscr{W} \mathscr{C}$ may be added to the list, but this fact is not required [7]. The separability assumption on each of the Banach spaces $E_{B_{n}}$ avoids the necessity of invoking Prokhorov's theorem. Since there exist $\sigma$-additive probabilities on Banach spaces which are not even scalarly concentrated on weakly compact sets, the separability of each space $E_{B_{n}}$ cannot be omitted. Furthermore, any Banach space is the union of its finite dimensional subspaces, 
so the countability of the family $\left(B_{n}\right)_{n \in N}$ is an essential condition.

Given any cylindrical probability $\mu$ on a locally convex space $E$, it is not hard to see that there exists a net of cylindrical probabilities with compact finite dimensional support converging to $\mu$ in the cylindrical topology. Such cylindrical probabilities have $(\tau, 1)$-moments, so convergence in $\gamma$ is not sufficiently restrictive to be of much use for investigating regularity.

However, if a condition is imposed on the additivity of the approximating net thereby limiting its size, convergence in the cylindrical topology is no longer vacuous.

Definition 3.2. Let $E$ be a locally convex space. A family $A \subseteq P(E)$ of cylindrical probabilities is called uniformly cylindrically additive if for each increasing sequence $\left(U_{n}\right)_{n \in N}$ of open cylinder sets such that $\lim _{n \in N} \mu\left(U_{n}\right)$ $=1$ for every $\mu \in A$, we have $\sup \left\{\mu\left(E \backslash U_{n}\right): \mu \in A\right\} \rightarrow 0$ as $n \rightarrow \infty$.

The following elementary observation is the basis of many of the applications of uniform cylindrical additivity.

Proposition 3.3. Let $A \subset \mathrm{P}(E)$ be a uniformly cylindrically additive family of cylindrical probabilities on the locally convex space $E$. Let $u$ : $E \rightarrow F$ be a continuous linear map into the locally convex space $F$.

Then the family $\left\{\mu \circ u^{-1}: \mu \in A\right\} \subset P(F)$ is uniformly cylindrically additive.

The next statements provide some conditions for a family of cylindrical probabilities to be uniformly cylindrically additive.

Proposition 3.4. A relatively $\sigma_{c}$-compact subset of $\mathrm{P}(E)$ is uniformly cylindrically additive.

Proof. Let ca $\left(\mathscr{C} \mathscr{L}\left(E^{*}\right)\right)$ be the space of countably additive set functions on $\mathscr{C} \mathscr{L}\left(E^{\prime *}\right)$ with the topology of setwise convergence. If $A \subset \mathrm{P}(E)$ is relatively $\sigma_{c}$-compact, then $\left\{\mu_{\sigma}: \mu \in A\right\}$ is a relatively compact subset of $\operatorname{ca}\left(\mathscr{C} \mathscr{L}\left(E^{\prime *}\right)\right)$.

According to a result of Bartle, Dunford and Schwartz (Dunford and Schwartz [4] p. 305), the family $\left\{\mu_{o}: \mu \in A\right\}$ is uniformly countably additive. Now suppose that $U_{n}, n=1,2, \cdots$ is an increasing family of open cylinder sets in $E$ such that $\mu\left(U_{n}\right) \rightarrow 1$ for every $\mu \in A$ as $n \rightarrow \infty$.

For each $n \in N$, let $\left(U_{n}\right)_{\sigma}$ be the open cylinder set in $E^{\prime *}$ induced by $U_{n}$. Then $\mu_{\sigma}\left(\left(U_{n}\right)_{\sigma}\right)=\mu\left(U_{n}\right)$ for every $n \in N$. For each $\mu \in A$. 


$$
\mu_{\sigma}\left[\left(\cup_{m \in N}\left(U_{m}\right)_{\sigma}\right] \backslash\left(U_{m}\right)_{\sigma}\right) \rightarrow 0
$$

as $n \rightarrow \infty$ and since $\left\{\mu_{\sigma}: \mu \in A\right\}$ is uniformly countably additive,

$$
\begin{aligned}
\sup \left\{\mu\left(E \backslash U_{n}\right): \mu \in A\right\} & =\sup \left\{\mu_{\sigma}\left(E^{\prime *} \backslash\left(U_{n}\right)_{\sigma}\right): \mu \in A\right\} \\
& =\sup \left\{\mu_{\sigma}\left(\bigcup_{m \in N}\left(U_{m}\right)_{\sigma} \backslash\left(U_{n}\right)_{\sigma}\right)\right\} \rightarrow 0
\end{aligned}
$$

as $n \rightarrow \infty$. Thus, the family $A$ is uniformly cylindrically additive.

In particular, if $\mu_{n}, n=1,2, \cdots$ is a sequence in $\mathrm{P}(E)$ converging in the topology $\sigma_{c}$ to $\mu \in \mathrm{P}(E)$, then the family $\left\{\mu_{n}: n \in N\right\}$ is uniformly cylindrically additive by the Vitali-Hahn-Saks theorem (Dunford and Schwartz [4] p. 159).

LEMMA 3.5. Let $Z \in \mathscr{Z}(E)$ be closed cylinder set. Then the map $\mu \rightarrow$ $\mu(Z), \mu \in \mathrm{P}(E)$ is upper semicontinuous on $(\mathrm{P}(E), \gamma)$.

Proof. For some continuous linear map $\phi: E \rightarrow R^{k}, k \in N$, and some closed set $C \subset R^{k}, Z=\phi^{-1}(C)$. The space $\left(\mathrm{P}\left(R^{k}\right), \gamma\right)$ is the space of regular Borel probabilities on $\boldsymbol{R}^{k}$, with the topology of convergence on bounded continuous functions. The map $\nu \rightarrow \nu(C), \nu \in \mathrm{P}\left(\boldsymbol{R}^{k}\right)$ is upper semicontinuous for $\gamma$ [1]. Furthermore, the map $\mu \rightarrow \mu \circ \phi^{-1}, \mu \in \mathrm{P}(E)$ is continuous between $(\mathrm{P}(E), \gamma)$ and $\left(\mathrm{P}\left(R^{k}\right), \gamma\right)$.

Thus, the map $\mu \rightarrow \mu(Z), \mu \in \mathrm{P}(E)$ is the composition of a continuous map and an upper semicontinuous map, so it is upper semicontinuous itself.

Theorem 3.6. Let $E$ be a locally convex space. A subset $A$ of $\mathrm{P}(E)$ is uniformly cylindrically additive if and only if the following two conditions hold.

(i) $A$ is relatively $\gamma$-compact.

(ii) If $F \in \mathscr{C} \mathscr{L}\left(E^{*}\right)$ and $\nu_{\sigma}(F)=1$ for every $\nu \in A$, then $\mu_{\sigma}(F)=1$ for every cylindrical probability $\mu$ belonging to the closure $\bar{A}^{r}$ of $A$ in the cylindrical topology $\gamma$.

Proof. Suppose that $A$ satisfies conditions (i) and (ii). Let $\left(U_{n}\right)_{n \in N}$ be an increasing sequence of open cylinder sets such that $\mu\left(U_{n}\right) \rightarrow 1$ as $n \rightarrow \infty$ for every $\mu \in A$. We show that $A$ is uniformly cylindrically additive by showing that the convergence is uniform for $\mu \in A$. For each $n \in N$, let $\left(U_{n}\right)_{\sigma} \in \mathscr{L}\left(E^{\prime *}\right)$ be the open cylinder set in $E^{\prime *}$ induced by $U_{n} \in$ $\mathscr{Z}(E)$. 
Define $\Phi_{n}: \mathrm{P}(E) \rightarrow[0,1]$ by $\Phi_{n}(\mu)=\mu\left(E \backslash U_{n}\right), \mu \in \mathrm{P}(E)$, for each $n \in N$. Now $\nu_{\sigma}\left(\cup_{n \in N}\left(U_{n}\right)_{\sigma}\right)=1$ for each $\nu \in A$, so condition (ii) implies that $\Phi_{n}$ decreases pointwise to zero on $\bar{A}^{r}$.

By Lemma 3.5, each map $\Phi_{n}$ is upper semicontinuous on $(\mathrm{P}(E), \gamma)$. Condition (i) together with Dini's theorem shows that $\Phi_{n} \rightarrow 0$ uniformly on $\bar{A}^{r}$. Thus, $\bar{A}^{r}$ is uniformly cylindrically additive.

Assume now that $A$ is uniformly cylindrically additive. To see first that it is a relatively $\gamma$-compact subset of $\mathrm{P}(E)$, we observe that it is an equicontinuous subset of the dual $M$ of $\mathrm{C}_{c}(E)$ equipped with the sup-norm. By the Banach-Alaoglu theorem, $A$ is a relatively $\sigma\left(M, \mathrm{C}_{c}(E)\right)$-compact subset of $M$. It suffices to show that the closure of $A$ in $\left(M, \sigma\left(M, \mathrm{C}_{c}(E)\right)\right)$ is contained in $\mathrm{P}(E)$.

Suppose that $\nu: \mathrm{C}_{c}(E) \rightarrow \boldsymbol{R}$ lies in the closure of $A$ (considered as linear functionals on $\left.\mathrm{C}_{c}(E)\right)$ in $\sigma\left(M, \mathrm{C}_{\mathrm{c}}(E)\right)$ ). Then $\nu$ defines the functional $\nu_{\sigma}: \mathrm{C}_{\sigma}\left(E^{\prime *}\right) \rightarrow R$ in an unambiguous manner. If $f_{n}, n=1,2, \cdots$ is a decreasing sequence of bounded continuous cylinder functions on $E^{\prime *}$ such that $f_{n} \rightarrow 0$ as $n \rightarrow \infty$, then the uniform cylindrical additivity of $A$ implies that $\nu_{\sigma}\left(f_{n}\right) \rightarrow 0$ as $n \rightarrow \infty$. Consequently, $\nu \in \mathrm{P}(E)$ so $A$ is relatively $\gamma$ compact.

Now suppose that $F \in \mathscr{C} \mathscr{L}\left(E^{\prime *}\right)$ and $\nu_{\sigma}(F)=1$ for every $\nu \in A$. Let $\mu$ be a cylindrical probability belonging to $\bar{A}^{r}$. We want to show that $\mu_{\sigma}(F)=1$.

Firstly,

$$
\begin{aligned}
\mu_{\sigma}(F) & =\inf \left\{\sum_{n \in N} \mu_{\sigma}\left(A_{n}\right): F \subset \bigcup_{n \in N} A_{n}, A_{n} \in \mathscr{Z}\left(E^{\prime *}\right)\right\} \\
& =\inf \left\{\sum_{n \in N} \mu_{\sigma}\left(U_{n}\right): F \subset \bigcup_{n \in N} U_{n}, U_{n} \in \mathscr{Z}\left(E^{\prime *}\right) \text { is open }\right\} \\
& =\inf \left\{\mu_{\sigma}\left(\bigcup_{n \in N} U_{n}\right): F \subset \bigcup_{n \in N} U_{n}, U_{n} \in \mathscr{Z}\left(E^{\prime *}\right) \text { is open }\right\} .
\end{aligned}
$$

Now each open set $U_{n} \in \mathscr{Z}\left(E^{\prime *}\right)$ is the union of a countable collection $\left(V_{m, n}\right)_{m \in N}$ of open cylinder sets such that $\bar{V}_{m, n} \subset U_{n}, m \in N$. If $F \subset \cup_{n \in N} U_{n}$, then for every $\varepsilon>0$, there are numbers $N, M \in N$ such that $\nu\left(\cup_{n \leqq N} \cup_{m \leqq M}\right.$ $\left.V_{m, n}\right) \geqq 1-\varepsilon$ for every $\nu \in A$, because $A$ is uniformly cylindrically additive.

There exists a net $\left(\mu_{i}\right)_{i \in I} \subset A$ such that $\mu_{i} \rightarrow \mu$ in the cylindrical topology $\gamma$. It follows from Badrikian [1] that

$$
\begin{aligned}
\mu_{\sigma}\left(\bigcup_{n \in N} U_{n}\right) & =\mu_{\sigma}\left(\bigcup_{n \in N} \bigcup_{m \in N} \bar{V}_{m, n}\right) \geqq \mu\left[\bigcup_{n \leqq N} \bigcup_{m \leqq M} \bar{V}_{m, n}\right] \\
& \geqq \lim _{i \in I} \sup \mu_{i}\left(\bigcup_{n \leqq N} \bigcup_{m \leqq M} \bar{V}_{m, n}\right) \geqq 1-\varepsilon,
\end{aligned}
$$


for each $\varepsilon>0$. Hence $\mu_{\sigma}(F)=1$ and condition (ii) is satisfied.

CoRollary 3.7. Let $\mathscr{B}$ be a complete convex bornology in the locally convex space $E$. Let $A$ be a collection of cylindrical probabilities on $E$ satisfying the following two conditions.

(i) A is uniformly cylindrically additive.

(ii) There exists a family of separable Banach spaces $\left(E_{B_{n}}\right)_{n \in N}$ with $B_{n} \in \mathscr{B}, n=1,2, \cdots$ such that $\nu_{\sigma}^{*}\left(\cup_{n \in N} E_{B_{n}}\right)=1$ for every $\nu \in A$.

If $\mu \in \bar{A}^{r}$, then $\mu$ extends to a unique $\mathscr{B} \mathscr{C}$-regular Borel measure on $F_{\beta}$,

Proof. Each of the disked sets $B_{n} \in \mathscr{B}, n \in N$ may be assumed to be closed in $E$. If $F \in \mathscr{C} \mathscr{L}\left(E^{\prime *}\right)$ and $\cup_{n \in N} E_{B_{n}} \subset F$, then $\nu_{o}(F)=1$ for each $\nu \in A$. By Theorem 3.6, $\mu_{\sigma}(F)=1$ also, so $\mu_{\sigma}^{*}\left(\cup_{n \in N} E_{B_{n}}\right)=1$. Now Lemma 3.1 may be applied.

The purpose of the assumption of uniform cylindrical additivity is to ensure that probabilities in the closure of a family in the cylindrical topology are concentrated on the same sets on which members of the original family are concentrated. For the topology $\sigma_{c}$, this holds automatically.

If $A$ is a relatively $\sigma_{c}$-compact subset of $\mathrm{P}(E)$, then the induced family of cylindrical probabilities $\left\{\mu_{\sigma}: \mu \in A\right\}$ on $E^{\prime *}$ is uniformly countably additive. By analogy, it might be imagined that a relatively $\gamma$-compact subset of $\mathrm{P}(E)$ is uniformly cylindrically additive; that is, condition (ii) follows from (i) in Theorem 3.6. The following example shows that this is not the case.

ExAMPLE 3.8. Let $\lambda: \mathscr{B} \mathcal{O}[0,1] \rightarrow[0,1]$ be the Lebesgue measure on $[0,1]$. Now define the vector-valued measure $m: \mathscr{B} \mathcal{O}[0,1] \rightarrow L^{1}(\lambda)$ by $m(A)$ $=\chi_{A}, A \in \mathscr{B} \mathcal{O}[0,1]$. Then the cylindrical probability $\nu$ associated with $(m, \lambda)$ (i.e. $\nu \circ \phi^{-1}=\lambda \circ(d \phi \circ m / d \lambda)^{-1}$ for all $\phi: E \rightarrow R^{k}, k \in N$ continuous and linear) has $(\tau, 1)$-moments, but it is not $\sigma$-additive on $L^{1}(\lambda)$, for otherwise $m$ would have a density with respect to $\lambda$, which is certainly not the case [3]. The random linear function associated with $\nu$ is just the natural inclusion $M: L^{\infty}(\lambda) \rightarrow L^{1}(\lambda)$.

Now let $u: \mathrm{C}[0,1] \rightarrow L^{1}(\lambda)$ be the inclusion map of the space of continuous functions $\mathrm{C}[0,1]$ on $[0,1]$ in $L^{1}(\lambda)$. For each $\theta \in \mathrm{C}[0,1]^{\prime}$, denote by $\theta_{\lambda}$ the $\lambda$-absolutely continuous part of the measure $\theta$. Let $\mu \in \mathrm{P}(\mathrm{C}[0,1])$ be the cylindrical probability defined by the linear random function $\theta \rightarrow$ $d \theta_{\lambda} / d \lambda \in L^{1}(\lambda), \theta \in \mathrm{C}[0,1]^{\prime}$. Then $\mu$ obviously has $(\beta, 1)$-moments. The com- 
position of the function $\theta \rightarrow d \theta_{\lambda} / \lambda, \theta \in \mathrm{C}[0,1]^{\prime}$ with the dual map $u^{\prime}: L^{\infty}(\lambda)$ $\rightarrow \mathrm{C}[0,1]^{\prime}$ is the linear random function associated with $\nu=\mu \circ u^{-1}$.

Thus, we have a cylindrical probability $\mu$ with $(\beta, 1)$-moments defined on the space of continuous function $\mathrm{C}[0,1]$, and a map $u: \mathrm{C}[0,1] \rightarrow L^{1}(\lambda)$ (which is absolutely summing) such that $\nu=\mu \circ u^{-1}$. Now it is proved in Schwartz [11] that there is a net $\left(\mu_{i}\right)_{i \in I}$ of Radon probabilities on C[0,1] with finite support such that $\mu_{i} \rightarrow \mu$ in the cylindrical topology on $\mathrm{P}(\mathrm{C}[0,1])$. Moreover, there exists a number $M>0$ such that for every $\xi \in \mathrm{C}[0,1]$, $\mu_{i}(|\xi|) \leqq M\|\xi\|$ for every $i \in I$.

Denote the bidual $L^{1}(\lambda)^{\prime \prime}$ for $L^{1}(\lambda)$ with the $\sigma\left(L^{1}(\lambda)^{\prime \prime}, L^{\infty}(\lambda)\right)$-topology by $F$. Let $\tilde{\nu}$ be the cylindrical probability induced by $\nu$ on $F$. For each $i \in I$, let $\tilde{\nu}_{i}$ be the cylindrical probability induced by $\nu_{i}=\mu_{i} \circ u^{-1}$ on $F$.

Given this somewhat elaborate construction, we can now describe the phenomenon. According to Schwartz [11], $\left(\tilde{\nu}_{i}\right)_{i \in I}$ is a relatively $\gamma$-compact family of cylindrical probabilities on $F$ such that $\tilde{\nu}_{i} \rightarrow \tilde{\nu}$ in $\gamma$. Furthermore, each $\tilde{\nu}_{i}, i \in I$ comes from a measure with finite support in $\mathrm{C}[0,1]$, so obviously $\left(\tilde{\nu}_{i}\right)_{\sigma}^{*}\left(L^{1}(\lambda)\right)=1$ for each $i \in I$. Nevertheless, we do not have $(\tilde{\nu})_{\sigma}^{*}\left(L^{1}(\lambda)\right)=1$, for otherwise $\nu$ would be $\sigma$-additive on $L^{1}(\lambda)$.

The problem is, of course, that $\left\{\tilde{\nu}_{i}: i \in I\right\}$ is not uniformly cylindrically additive on $F$, even though it is relatively $\gamma$-compact. In this example, it is true that $\tilde{\nu}$ is $\sigma$-additive on $F$. Furthermore, the net $\left(\tilde{\nu}_{i}\right)_{i \in I}$ can even be taken to be a sequence, because $\mathrm{C}[0,1]$ is a separable Banach space.

It is clear from Example 3.8 that the distinction between uniform cylindrical additivity and relative $\gamma$-compactness is of central importance for the technique of approximating cylindrical probabilities in the $\gamma$ topology.

A set $A \subset \mathrm{P}(E)$ is relatively compact in $\sigma_{c}$ if and only if it is uniformly cylindrically additive, and for each set $B \in \mathscr{Z}(E), \varepsilon>0$, there exists an open cylinder set $U \in \mathscr{Z}(E)$ such that $B \subset U$ and $\mu(B \backslash U)<\varepsilon$ for all $\mu \in A$. Indeed, if $A$ is uniformly cylindrically additive with the given uniform outer-regularity property, then it follows that $\left\{\mu_{\sigma}: \mu \in A\right\}$ is uniformly countably additive on $\mathscr{Z}\left(E^{\prime *}\right)$, from which follows relative $\sigma_{c}$-compactness. Conversely, any relatively $\sigma_{c}$-compact subset of $\mathrm{P}(E)$ is uniformly absolutely continuous with respect to some cylindrical probability on $E$, so the set $A \subset \mathrm{P}(E)$ has the uniform outer regularity property.

Thus, we see that uniform cylindrical additivity is stronger than relative compactness in the cylindrical topology $\gamma$, but weaker than relative 
$\sigma_{c}$-compactness by virtue of the absence of the condition of uniform outer regularity on $\mathscr{Z}(E)$.

\section{$\S 4$. Images by nuclear maps}

Suppose that $u: E \rightarrow F$ is a nuclear map between locally convex spaces $E$ and $F[10]$. Given a vector measure $m: \mathscr{S} \rightarrow E$ absolutely continuous with respect to a probability $\lambda$, it is a simple matter to verify that $u \circ m$ has a density $f$ with respect to $\lambda$ such that the probability $\lambda \circ f^{-1}$ has strong regularity properties.

In view of Corollary 3.7, cylindrical probabilities which lie in the $\gamma$ closure of a sufficiently small family of cylindrical probabilities with moments will be mapped into regular measures by nuclear maps. This basic idea allows us to provide a characterization of regular measures on conuclear spaces alternate to that of Minlos's theorem.

Call a subset $A$ of a locally convex space $E$ completing if $A$ is contained in a bounded, disked set $B$ for which the space $E_{B}$ is complete.

THEOREM 4.1. Let $E$ and $F$ be locally convex spaces and $u: E \rightarrow F a$ linear map for which $u=\sum_{n \in N} \alpha_{n} \xi_{n} \otimes y_{n}$, where $\sum_{n \in N}\left|\alpha_{n}\right|<\infty,\left\{\xi_{n}: n \in N\right\}$ is completing in $E_{\sigma}^{\prime}$, and $\left\{y_{n}: n \in N\right\}$ is completing in $F$.

Suppose that $\mu \in \mathrm{P}(E)$ has one of the following properties.

$\mathrm{N}_{1}$ : There exists a net $\left(\mu_{i}\right)_{i \in I} \subset \mathrm{P}(E)$ of cylindrical probabilities with $(\beta, 1)$-moments such that $\mu_{i} \rightarrow \mu$ in $\sigma_{c}$.

$\mathrm{N}_{2}$ : There exists a uniformly cylindrically additive net $\left(\mu_{i}\right)_{i \in I} \subset \mathrm{P}(E)$ of cylindrical probabilities with $(\beta, 1)$-moments such that $\mu_{i} \rightarrow \mu$ in $\gamma$.

Then $\mu \circ u^{-1}$ extends to a $\mathscr{B} \mathscr{C}$-regular Borel measure on $F_{\beta}$.

Proof. Assume first that $\nu \in \mathrm{P}(E)$ has $(\beta, 1)$-moments. Denote the identity on $E^{\prime *}$ by $\iota_{\sigma}$. Then $\iota_{\sigma}$ is $\nu_{\sigma}$-integrable in $E_{\sigma}^{\prime \prime}$; that is, $\iota_{\sigma} \nu_{\sigma}: \mathscr{C} \mathscr{L}\left(E^{\prime *}\right)$ $\rightarrow E_{\sigma}^{\prime \prime}$ is a vector measure.

Since the sequence $\left(\xi_{n}\right)_{n \in N}$ is completing, it is bounded for the strong topology $\beta\left(E^{\prime}, E\right)$ by the Banach-Mackey theorem (Schaefer [10] p. 194). An extension of $u$ to $E^{\prime \prime}$, still denoted by $u$, may be defined by $u\left(x^{\prime \prime}\right)=$ $\sum_{n \in N} \alpha_{n} x^{\prime \prime}\left(\xi_{n}\right) y_{n}, x^{\prime \prime} \in E^{\prime \prime}$. Since $\left(\xi_{n}\right)_{n \in N}$ is $\beta\left(E^{\prime}, E\right)$-bounded $\left(x^{\prime \prime}\left(\xi_{n}\right)\right)_{n \in N}$ is bounded for every $x^{\prime \prime} \in E^{\prime \prime}$, and the series converges in $F$ because $\left(y_{n}\right)_{n \in N}$ is completing.

Now $\iota_{\sigma} \nu_{\sigma}\left(\mathscr{C} \mathscr{L}\left(E^{*}\right)\right)$ is a relatively weakly compact, and so strongly bounded subset of $E_{\sigma}^{\prime \prime}$. Since $\left(\xi_{n}\right)_{n \in N}$ is $\beta\left(E^{\prime}, E\right)$-bounded it follows that 
$\left\{\left\langle\iota_{\sigma} \nu_{\sigma}(A), \xi_{n}\right\rangle: A \in \mathscr{C} \mathscr{L}\left(E^{*}\right), n \in N\right\}$ is a bounded subset of $\boldsymbol{R}$. For every $\zeta \in F^{\prime},\left\langle u \circ \iota_{\sigma} \nu_{\sigma}, \zeta\right\rangle=\sum_{n \in N} \alpha_{n}\left\langle\iota_{\sigma} \nu_{\sigma}, \xi_{n}\right\rangle\left\langle y_{n}, \zeta\right\rangle$ is a measure by the dominated convergence, so $u \circ \iota_{\sigma} \mu_{\sigma}=\sum_{n \in N} \alpha_{n}\left\langle\iota_{\sigma} \nu_{\sigma}, \xi_{n}\right\rangle y_{n}$ is a vector measure in $F$ by the Orlicz-Pettis theorem.

The scalar version of the Radon-Nikodým theorem implies that there exists a sequence $f_{n}: E^{*} \rightarrow R, n=1,2, \cdots$ of $\mu_{\sigma}$-integrable functions such that $u \circ \iota_{\sigma} \nu_{\sigma}=\sum_{n \in N} \alpha_{n}\left(f_{n} \nu_{\sigma}\right) y_{n}$.

Now the sequence $\left(\nu_{\sigma}\left(\left|f_{n}\right|\right)\right)_{n \in N}$ is bounded, so $\sum_{n \in N}\left|\alpha_{n}\right|\left|f_{n}\right|<\infty \mu_{\sigma}$-a.e. by monotone convergence. Again, $\left(y_{n}\right)_{n \in N}$ is completing, so $f: E^{* *} \rightarrow F$ may be defined by $f(\omega)=\sum_{n \in N} \alpha_{n} f_{n}(\omega) y_{n}$ if $\sum_{n \in N}\left|\alpha_{n}\right|\left|f_{n}(\omega)\right|<\infty$ and 0 elsewhere. Then $u \circ \iota_{\sigma} \nu_{\sigma}=f \nu_{\sigma}$.

Since $\nu \circ u^{-1}$ is the cylindrical probability associated with $\left(u \circ c_{\sigma} \nu_{\sigma}, \nu_{\sigma}\right)$, the equality $\nu \circ u^{-1}=\nu_{\sigma} \circ f^{-1}$ holds on $\mathscr{Z}(E)$. Thus, the sequence $\left(y_{n}\right)_{n \in N}$ is contained in a separable Banach space $G$ included in $F$ such that $\left(\nu \circ u^{-1}\right)_{\sigma}^{*}(G)=1$.

For the general case when there exists a net $\left(\mu_{i}\right)_{i \in I}$ satisfying conditions $\left(\mathrm{N}_{1}\right)$ or $\left(\mathrm{N}_{2}\right)$, the Banach space $G$ included in $F$ is fixed, since it was constructed from $u$, and we have $\left(\mu_{i} \circ u^{-1}\right)_{\sigma}^{*}(G)=1$ for all $i \in I$. The conclusion now follows from Corollary 3.7 and Proposition 3.3.

The basic idea of this proof is quite common in the literature (Bradrikian [1], Chi [2]), and with suitable modifications, an analogous assertion holds for $(\beta, p)$-moments, $p \geqq 1$.

Theorem 4.1 may be viewed as an extension of the result of L. Schwartz [11], that nuclear maps between Banach spaces are 1-radonifying. Substantially different techniques are used in [11].

Recall that a nuclear map $u: E \rightarrow F$ is defined just as in Theorem 4.1 except that the family $\left(\xi_{n}\right)_{n \in N}$ is assumed to be equicontinuous in $E^{\prime}$, which is more than enough to ensure that it is completing.

We will call a continuous linear map $u: E \rightarrow F$ quasinuclear, if there exists a completing disked set $B \cup F$, and a disked neighbourhood $U$ of zero in $E$, such that $u$ admits a factorization $u=\psi_{B} \circ u_{0} \circ \phi_{U}$, where $\psi_{B}$ : $F_{B} \rightarrow F$ and $\phi_{U}: E \rightarrow E_{U}$ are the natural maps, and $u_{0}: \hat{E}_{U} \rightarrow F_{B}$ is quasinuclear between the completion $\hat{E}_{U}$ of $E_{U}$ and $F_{B}$; that is, $p_{B}\left(u_{0}(x)\right) \leqq$ $\sum_{n \in N}\left|a_{n} \| \xi_{n}(x)\right|, x \in E_{U}$, for some sequence $\left(a_{n}\right)_{n \in N} \in \ell^{1}$, and $\left(\xi_{n}\right)_{n \in N}$ bounded in $\left(E_{U}\right)^{\prime}[8]$ 3.2.3.

A characterization of quasinuclear maps established by A. Pietsch [8] 3.2.6, yields the following consequence of Theorem 4.1. 
Corollary 4.2. Let $E$ and $F$ be locally convex spaces and let $u: E \rightarrow F$ be a quasinuclear map. Suppose that the cylindrical probability $\mu \in \mathrm{P}(E)$ satisfies either condition $\left(\mathrm{N}_{1}\right)$ or $\left(\mathrm{N}_{2}\right)$ of Theorem 4.1 .

Then $\mu \circ u^{-1}$ extends to a $\mathscr{B}_{0} \mathscr{C}$-regular Borel measure on $F_{\beta}$.

Theorem 4.1 and Corollary 4.2 give a class of cylindrical probabilities which are regularized by nuclear maps between locally convex spaces. There is therefore some resemblance with the theorem of Sazanov which asserts that a continuous linear map $u: E \rightarrow F$ between Hilbert spaces $E$ and $F$ is Hilbert-Schmidt if and only if for each $\mu \in \mathrm{P}(E)$ scalarly concentrated on bounded sets (respectively, the canonical Gauss measure $\gamma$ ), the cylindrical probability $\mu \circ u^{-1}$ (respectively, $\gamma \circ u^{-1}$ ) is cylindrically concentrated on bounded sets in $F$ (Schwartz [13]).

To give examples of cylindrical probabilities on some locally convex space which are scalarly concentrated on weakly compact sets, but which do not have the approximation properties $\mathrm{N}_{1}$ or $\mathrm{N}_{2}$, we appeal to another result of L. Schwartz.

Example 4.3. Let $E$ be the space $\ell^{\infty}$ endowed with its weak* topology $\sigma\left(l^{\infty}, l^{1}\right)$. Let $F$ be the space $\ell^{1}$ with its weak topology $\sigma\left(\ell^{1}, \ell^{\infty}\right)$. Define the map $u: E \rightarrow F$ by

$$
u\left(\left(x_{n}\right)_{n \in N}\right)=\left(1 /\left(n \log ^{2}(n+1)\right) x_{n}\right)_{n \in N}, \quad\left(x_{n}\right)_{n \in N} \in \ell^{\infty} .
$$

Then

$$
\sum_{n \in N} 1 /\left(n \log ^{2}(n+1)\right)<\infty,
$$

so that $u$ is certainly a map of the type described in Theorem 4.1. However, we also have

$$
\sum_{n \in N} 1 /\left(n \log ^{2}(n+1)\right) \log \left(n \log ^{2}(n+1)\right)=\infty,
$$

so from Schwartz [13] p. 347, there is a cylindrical probability $\mu \in \mathrm{P}(E)$ scalarly concentrated on bounded sets such that $\mu \circ u^{-1}$ is not $\sigma$-additive on $\ell^{1}$.

According to Theorem 4.1, $\mu$ cannot have either of the properties $\mathrm{N}_{1}$ and $\mathrm{N}_{2}$.

It could be argued that cylindrical probabilities which do not have the approximation properties $\mathrm{N}_{1}$ or $\mathrm{N}_{2}$ are mere curiosities, more so than those whose Fourier transforms are not continuous. Indeed, it is easier 
to find examples of cylindrical probabilities satisfying $N_{1}$ and $N_{2}$ than it is to find examples of those which do not.

Proposition 4.4. Let $\mu$ be a cylindrical probability on the locally convex space $E$ such that $\mu$ is cylindrically concentrated on bounded subsets of $E$.

Then there exists a sequence $\mu_{n} \in \mathrm{P}(E), n=1,2, \cdots$ such that for each $n \in N, \mu_{n}$ has $(\beta, 1)$-moments and $\mu_{n} \rightarrow \mu$ in the topology $\sigma_{c}$ as $n \rightarrow \infty$. In particular, $\mu$ satisfies both conditions $\mathrm{N}_{1}$ and $\mathrm{N}_{2}$.

Proof. Let $B_{n}, n=1,2, \cdots$ be bounded subsets of $E$ for which

$$
\left[\mu \circ \phi^{-1}\right]^{*}\left(\phi\left(B_{n}\right)\right) \geqq 1-1 / 2 n, \quad n=1,2, \cdots
$$

for all $\phi \in \mathscr{L}\left(E, R^{k}\right), k \in N$.

Let $\nu$ be the cylindrical probability induced by $\mu$ on $E_{\sigma}^{\prime \prime}$; that is, identifying $E$ with its canonical image in $E^{\prime \prime}$,

$$
\nu(A)=\mu(A \cap E), \quad A \in \mathscr{Z}\left(E_{\sigma}^{\prime \prime}\right) .
$$

Then $\nu$ is cylindrically concentrated on the closures $\bar{B}_{n}$ of $B_{n}$ in $E_{\sigma}^{\prime \prime}$, $n=1,2, \cdots$, so by Prokhorov's theorem [13], it extends to a Radon measure on $E_{\sigma}^{\prime \prime}$, denoted again by $\nu$.

Define $\mu_{n} \in \mathrm{P}(E)$ by

$$
\mu_{n}(A \cap E)=\nu\left(A \cap \bar{B}_{n}\right) / \nu\left(\bar{B}_{n}\right), \quad A \in \mathscr{Z}\left(E_{\sigma}^{\prime \prime}\right)
$$

for $n=1,2, \cdots$. Then

$$
\mu_{n}(|\xi|) \leqq \sup \left\{|\langle x, \xi\rangle|: x \in B_{n}\right\}, \quad \xi \in E^{\prime}, \quad n=1,2, \cdots
$$

so each $\mu_{n}$ certainly has $(\beta, 1)$-moments. It is easily verified that $\mu_{n} \rightarrow \mu$ in $\sigma_{c}$ as $n \rightarrow \infty$, proving the result.

Corollary 4.5. Let $E$ be a locally convex space which is the union of a countable system of bounded subsets.

If $\mu \in \mathrm{P}(E)$ is $\sigma$-additive, then there exists a sequence $\mu_{n}, n=1,2, \cdots$, of cylindrical probabilities with $(\beta, 1)$-moments on $E$ such that $\mu_{n} \rightarrow \mu$ in $\sigma_{c}$.

Proof. Let $B_{n}, n=1,2, \cdots$ be an increasing family bounded subsets of $E$ such that $E=\cup_{n \in N} B_{n}$. Then

$$
\mu^{*}(E)=\lim _{n \rightarrow \infty} \mu^{*}\left(B_{n}\right)=1,
$$

so that $\mu$ is cylindrically concentrated on the family $\left\{B_{n}: n=1,2, \cdots\right\}$ 
of subsets of $E$. Now appeal to the preceding assertion.

Thus, $\sigma$-additive cylindrical probabilities on Banach spaces satisfy conditions $\mathrm{N}_{1}$ and $\mathrm{N}_{2}$, but it is well-known that they need not be scalarly concentrated on weakly compact sets. The conditions $\mathrm{N}_{1}$ and $\mathrm{N}_{2}$ that a cylindrical probability is approximable by cylindrical probabilities with moments are therefore quite general.

Theorem 4.1 may now be applied to a conuclear space, whose internal structure is derived from nuclear maps.

Let $\mathscr{B}$ be a convex bornology in the locally convex space $E$. We say that $\mathscr{B}$ is conuclear if each closed, disked set $A \in \mathscr{B}$ is contained in another closed, disked set $B \in \mathscr{B}$ such that the natural injection $j: E_{A} \rightarrow E_{B}$ of the completion of $E_{A}$ into the completion of $E_{B}$ is nuclear [13].

TheOREm 4.6. Let $\mathscr{B}$ be a complete, conuclear, convex bornology in the locally convex space $E$.

Then $\mu \in \mathrm{P}(E)$ extends to a $\mathscr{B} \mathscr{C}$-regular Borel measure on $F_{\beta}$ if and only if one of the following conditions holds.

(i) There exists a countable net $\left(\mu_{i}\right)_{i \in I} \subset \mathrm{P}(E)$ of cylindrical probabilities with $(\mathscr{B}, 1)$-moments such that $\mu_{i} \rightarrow \mu$ in $\sigma_{c}$.

(ii) There exists a countable, uniformly cylindrically additive net $\left(\mu_{i}\right)_{i \in I} \subset \mathrm{P}(E)$ of cylindrical probabilities with $(\mathscr{B}, 1)$-moments such that $\mu_{i} \rightarrow \mu$ in $\gamma$.

Proof. Nuclear maps send bounded sets into relatively compact sets, so if $\mathscr{B}$ is conuclear and complete, then $\mathscr{B}=\mathscr{B} \mathscr{W} \mathscr{C}=\mathscr{B} \mathscr{C}$.

Suppose first that $\nu \in \mathrm{P}(E)$ has $(\mathscr{B}, 1)$-moments. Then $\nu$ obviously has $(\tau, 1)$-moments and there exists a closed disked set $A \in \mathscr{B}$ such that $\iota_{\sigma} \nu_{\sigma}$ : $\mathscr{C} \mathscr{L}\left(E^{\prime *}\right) \rightarrow E_{A}$ is a vector measure. Furthermore, there exists a closed, disked set $B \in \mathscr{B}$ such that $j: E_{A} \rightarrow E_{B}$ is nuclear.

The cylindrical probability associated with the pair $\left(\iota_{\sigma} \nu_{\sigma}, \nu_{\sigma}\right)$ on $E_{A}$ factors through $E_{B}$ onto $E$, and it is easy to see that the result is $\nu$. From Theorem 4.1, it follows that $\nu_{\sigma}^{*}\left(E_{B}\right)=1$.

The general case now follows from Lemma 3.1, Proposition 3.3, and Corollary 3.7.

Conversely, if $\mu \in \mathrm{P}(E)$ extends to a $\mathscr{B} \mathscr{C}$-regular Borel measure $\nu$ on $E$, then the normalized restrictions of $\nu$ to a suitable increasing family of $\mathscr{B}$-strictly compact sets yields the desired family of cylindrical probabilities. 
According to the celebrated result of Minlos [13] p. 233, a cylindrical probability on a locally convex space with a complete, conuclear, convex bornology $\mathscr{B}$, extends to a $\mathscr{B}$-regular Radon measure on $E$ if and only if it is scalarly concentrated on the family $\mathscr{B}$.

Thus, it appears a posteriori, that the class of cylindrical probabilities which are scalarly concentrated on $\mathscr{B}$ coincides with that class possessing the approximating property of Theorem 4.6 whenever $\mathscr{B}$ is conuclear. Example 4.3 shows that this equivalence is false in general. It is not known whether there are bornologies other than conuclear ones for which these classes coincide.

The introduction of generalized stochastic processes (random linear functions) and cylindrical probabilities was initially related to problems arising in quantum field theory. Some of the current techniques employ approximations by constructions on the discrete space-time lattice as the lattice spacing tends to zero. In this manner, the Euclidean quantum field measure is obtained as a limit of the simpler lattice fields measures: a technique reminiscent of Theorem 4.6 (see, for example, Glimm and Jaffe [5]).

\section{§. Images by absolutely summing maps and the Radon-Nikodým Theorem}

The theme of the preceding section can be continued with absolutely summing maps in place of nuclear maps. Again, the technique used is to exploit the properties of the vector measure associated with a cylindrical probability with $(\tau, 1)$-moments. The relevant methods have been developed by E. Thomas [14] for vector-valued Radon measures, but the same argument applies to abstract vector measures as well.

First, a few notions from [8] are needed. Let $E$ be a locally convex space. A family $\left(x_{i}\right)_{i \in I} \subset E$ is said to be summable if the family of finite partial sums forms a directed Cauchy system. Now let $\mathscr{U}$ be a fundamental system of disked neighbourhoods of zero in $E$. A family $\left(x_{i}\right)_{i \in I} \subset E$ is called absolutely summable if

$$
\sum_{i \in I} p_{U}\left(x_{i}\right)<\infty
$$

for all $U \in \mathscr{U}$.

A continuous linear map is said to be absolutely summing if it maps summable sequences into absolutely summable sequences. 
Let $\ell^{1}(E)$ be the family of sequences $\left(x_{n}\right)_{n \in N} \subset E$ such that

$$
\sum_{n \in N}\left|\left\langle x_{n}, \xi\right\rangle\right|<\infty, \quad \xi \in E^{\prime} .
$$

For each $U \in \mathscr{U}$, denote by $\varepsilon_{U}$ the seminorm defined by

$$
\varepsilon_{U}\left(\left(x_{n}\right)_{n \in N}\right)=\sup \left\{\sum_{n \in N}\left|\left\langle x_{n}, \xi\right\rangle\right|: \xi \in U^{\circ}\right\}
$$

for every $\left(x_{n}\right)_{n \in N} \in \ell^{1}(E)$. The space $\ell^{1}(E)$ is assumed to be equipped with the locally convex topology defined by the family $\left\{\varepsilon_{U}: U \in \mathscr{U}\right\}$ of seminorms.

Similarly, given $\ell^{1}\{E\}$, the space of absolutely summable sequences in $E$, the topology defined by the family $\left\{\pi_{U}: U \in \mathscr{U}\right\}$ of seminorms, where

$$
\pi_{U}\left(x_{n}\right)_{n \in N}=\sum_{n \in N} p_{U}\left(x_{n}\right), \quad\left(x_{n}\right)_{n \in N} \in \ell^{1}\{E\}
$$

for each $U \in \mathscr{U}$.

Then a continuous linear map $u: E \rightarrow F$ between locally convex spaces $E$ and $F$ is absolutely summable if and only if it maps bounded subsets of $\ell^{1}(E)$ into bounded subsets of $\ell^{1}\{E\}$ [8]. The definition of absolutely summing maps given by Thomas [14] is thereby only formally different from that of Pietsch [8].

For normed spaces, there is a useful characterization of these maps. Namely, let $u: E \rightarrow F$ be an absolutely summing map between the normed spaces $E$ and $F$. Let $U$ be the closed unit ball of $E^{\prime}$ and $\|\cdot\|$ the norm of $F$. Then there is a Radon measure $\nu$ on $U$ equipped with the relative $\sigma\left(E^{\prime}, E\right)$-topology such that $\|u(x)\| \leqq \nu(|\langle x, \cdot\rangle|)$ for every $x \in E$ [8] 2.3.3. It is clear that any map of this form is absolutely summing.

We first give a simplified proof of a result of L. Schwartz [11]. The original proof uses the approximation property for the space of continuous functions on a compact set, but it seems that vector measures lie at the heart of these matters.

Lemma 5.1. Let $E$ and $F$ be Banach spaces. If $u: E \rightarrow F$ is absolutely summing map, then $u$ extends uniquely to an absolutely summing map $u^{\prime \prime}$ : $\left(E^{\prime \prime}, \tau\left(E^{\prime \prime}, E^{\prime}\right)\right) \rightarrow F$.

Proof. There exists a Radon measure $\nu$ on the closed unit ball of $E^{\prime}$ such that

$$
\|u(x)\| \leqq \nu(\mid\langle x, \cdot\rangle), \quad x \in E .
$$

Let $j: E \rightarrow L^{\infty}(\nu)$ be the injection identifying each $x \in E$ with its equivalence 
class in $L^{\infty}(\nu)$. Then $j$ is $\beta\left(E, E^{\prime}\right)-\tau\left(L^{\infty}, L^{1}\right)$ continuous, and so the dual map $j^{\prime}: L^{1} \rightarrow E_{\beta}^{\prime}$ is continuous. Consequently, the bidual $j^{\prime \prime}: E^{\prime \prime} \rightarrow L^{\infty}$ is $\sigma\left(E^{\prime \prime}, E^{\prime}\right)$ $\sigma\left(L^{\infty}, L^{1}\right)$ continuous, and so $\tau\left(E^{\prime \prime}, E^{\prime}\right)-\tau\left(L^{\infty}, L^{1}\right)$ continuous [10] IV, 7.4.

Because the inclusion of $L^{\infty}(\nu)$ in $L^{1}(\nu)$ is $\sigma\left(L^{\infty}, L^{1}\right)-\sigma\left(L^{1}, L^{\infty}\right)$ continuous, it is $\tau\left(L^{\infty}, L^{1}\right)-\beta\left(L^{1}, L^{\infty}\right)$ continuous, so the map $u^{\prime \prime}: E^{\prime \prime} \rightarrow F$ can be defined by factoring it through $L^{\infty}(\nu)$ via the map $j^{\prime \prime}: E^{\prime \prime} \rightarrow L^{\infty}(\nu)$.

Furthermore, the inclusion map

$$
k:\left(L^{\infty}(\nu), \sigma\left(L^{\infty}, L^{1}\right)\right) \rightarrow L^{1}
$$

is absolutely summing because the inclusion of $L^{\infty}(\nu)$ in $L^{1}(\nu)$ is absolutely summing, and the unit ball of $L^{1}(\nu)$ is $\sigma\left(\left(L^{\infty}\right)^{\prime}, L^{\infty}\right)$-dense in the unit ball of $L^{\infty}(\nu)^{\prime}$ by Goldstine's theorem. The composition of a continuous and an absolutely summing map is certainly absolutely summing, so $u^{\prime \prime}$ is absolutely summing.

Theorem 5.2. Let $E$ and $F$ be Banach spaces. Let $u: E \rightarrow F$ be an absolutely summing map. If the cylindrical probability $\mu \in \mathrm{P}(E)$ has $(\beta, 1)$ moments, then $\mu \circ u^{-1}$ is cylindrically concentrated on bounded subsets of $F$.

Proof. If $\mu$ has $(\beta, 1)$-moments, then $\iota_{\sigma} \mu_{\sigma}: \mathscr{C} \mathscr{L}\left(E^{* *}\right) \rightarrow E_{\sigma}^{\prime \prime}$ is a vector measure with values in $E^{\prime \prime}$. Denoting the natural inclusion of $E$ into $E^{\prime \prime}$ by $j, \mu \circ j^{-1}$ is the cylindrical probability associated with the pair $\left(\iota_{\sigma} \mu_{\sigma}, \mu_{\sigma}\right)$ (see, for example [7]). Suppose that $u^{\prime \prime}: E^{\prime \prime} \rightarrow F$ is the absolutely summing extension of $u$ defined in Lemma 5.1 .

Since $u^{\prime \prime}: E_{\tau}^{\prime \prime} \rightarrow F$ is absolutely summing, it maps bounded subsets of $\ell^{1}\left(E_{\sigma}^{\prime \prime}\right)$ into bounded subsets of $\ell^{1}\{F\}$. In particular $u^{\prime \prime} \circ \iota_{\sigma} \mu_{\sigma}$ has bounded variation in $F$. According to [7] Theorem 3, the $\left(u^{\prime \prime} \circ \iota_{\sigma} \mu_{\sigma}, \mu_{\sigma}\right)$-distribution, $\left(\mu \circ j^{-1}\right) \circ u^{\prime \prime-1}$ is cylindrically concentrated on bounded sets in $F$. Since $u=u^{\prime \prime} \circ j$, the conclusion is immediate.

Similar arguments hold for $p$-summing maps and cylindrical probabilities with $(\beta, p)$-moments for $p>1$ [11]. In this case, the maps factor through an $L^{p}$-space, which is reflexive. It follows that the image measure is cylindrically concentrated on weakly compact sets, so it extends to a Radon measure.

By virtue of the relationship of vector measures with cylindrical probabilities with $(\tau, 1)$-moments, we have the following application of a result of E. Thomas [14] 4.1. The passage from vector-valued Radon measures to arbitrary vector measures presents no problem. 
Theorem 5.3. Let $E$ and $F$ be Banach spaces. Let $u: E \rightarrow F$ be an absolutely summing map. If $\mu \in \mathrm{P}(E)$ has $(\tau, 1)$-moments, then $\mu \circ u^{-1}$ extends to a Radon probability on $E$.

Again, bornologies afford a quick extension for the approximating case in locally convex spaces once the terms are defined appropriately.

Definition 5.4. Let $\mathscr{B}$ and $\mathscr{C}$ be convex bornologies in the locally convex spaces $E, F$ respectively.

A continuous linear map $u: E \rightarrow F$ is said to be $\mathscr{B}$ - $\mathscr{C}$-totally summing if for each disked set $B \in \mathscr{B}$, there exists a disked set $C \in \mathscr{C}$ such that $u(B) \subset C$, and $u: E_{B} \rightarrow E_{C}$ is absolutely summing.

If $\mathscr{B}$ and $\mathscr{C}$ are the bornologies of all bounded sets, then we merely say $u$ is totally summing.

The terminology is suggested by the term totally summable in Definitioy 1.5.1 of [8]. Moreover, a continuous linear map $u: E \rightarrow F$ is totally summing if and only if it sends bounded subsets of weakly summable families $\ell^{1}(E)$ into bounded totally summable families.

Quasinuclear maps between locally convex spaces are totally summing, because the same holds true in normed space [8] 3.2.13. Furthermore, if $F$ is a space with property (B) [8] 1.5 .5 , then $u \in \mathscr{L}(E, F)$ is totally summing if and only if it is absolutely summing.

A convex bornology $\mathscr{B}$ in the locally convex space $E$ is conuclear if and only if the identity on $E$ is $\mathscr{B}$ - $\mathscr{B}$-totally summing [8].

Theorem 5.5. Let $E$ and $F$ be locally convex spaces with complete convex bornologies $\mathscr{B}$ and $\mathscr{C}$ respectively. Let $u \in \mathscr{L}(E, F)$ be a $\mathscr{B}$ - $\mathscr{C}$-totally summing map. Suppose that $\mu \in \mathrm{P}(E)$ has one of the following properties.

(i) There exists a countable net $\left(\mu_{i}\right)_{i \in I} \subset \mathrm{P}(E)$ of cylindrical probabilities with $(\mathscr{B} \mathscr{W} \mathscr{C}, 1)$-moments such that $\mu_{i} \rightarrow \mu$ in $\sigma_{c}$.

(ii) There exists a countable, uniformly cylindrically additive net $\left(\mu_{i}\right)_{i \in I}$ $\subset \mathrm{P}(E)$ of cylindrical probabilities with $(\mathscr{B} \mathscr{W} \mathscr{C}, 1)$-moments such that $\mu_{i} \rightarrow \mu$ in $\gamma$.

Then $\mu \circ u^{-1}$ extends to a $\mathscr{B} \mathscr{C}$-regular Borel measure on $E_{\beta}$.

Proof. Consider first the case where $\nu \in \mathrm{P}(E)$ has $(\mathscr{B} \mathscr{W} \mathscr{C}, 1)$-moments. Then for some disked set $A \in \mathscr{B}, \iota_{\sigma} \nu_{\sigma}: \mathscr{C} \mathscr{L}\left(E^{\prime *}\right) \rightarrow E_{A}$ is a vector measure, and the $\left(\iota_{\sigma} \nu_{\sigma}, \nu_{\sigma}\right)$-distribution $\eta$ on $E_{A}$ has $(\tau, 1)$-mements. The image of $\eta$ by the inclusion of $E_{A}$ in $E$ is just $\nu$ itself. Since $u$ is $\mathscr{B}-\mathscr{C}$-totally summing, 
there exists a disked set $B \in \mathscr{C}$ such that $u(A) \subset B$ and $u: E_{A} \rightarrow E_{B}$ is absolutely summing.

According to Theorem 5.3, the $\left(u \circ c_{\sigma} \nu_{\sigma}, \nu_{\sigma}\right)$-distribution extends to a Radon measure on $F_{B}$, so there exists a closed, separable subspace $G$ of $E_{B}$ such that $\left(\nu \circ u^{-1}\right)_{\sigma}^{*}(G)=1$.

In general, there exists a countable family $F_{B_{i}}, i=I$ of separable Banach spaces with $B_{i} \in \mathscr{C}$ for $i \in I$ such that

$$
\left(\mu_{i} \circ u^{-1}\right)_{o}^{*}\left(F_{B_{i}}\right)=1, \quad i \in I .
$$

The conclusion now follows from Lemma 3.1, Corollary 3.7 and Proposition 3.3.

If the countability assumption is dropped, then Theorem 3.6 shows that we may still conclude that $\mu \circ u^{-1}$ is $\sigma$-additive. Nevertheless, a close look at Example 3.8 shows that uniform cylindrical additivity cannot also be omitted from the assumptions. A simple example illustrates the type of situation where these ideas are applicable.

ExAmple 5.6. Let $\lambda$ be the Lebesgue measure on $[0,1]$. Define $f$ : $[0,1] \rightarrow R^{[0,1]}$ by

$$
f(s)(t)=(s-t)^{-1 / 2}
$$

if $s, t \in[0,1], s \neq t$ and zero otherwise. Let $C_{s}[0,1]$ be the space of continuous functions on $[0,1]$ furnished with the topology of pointwise convergence.

Then the indefinite integral $f \lambda$ of $f$ with respect to $\lambda$ has its values in the space $\mathrm{C}_{s}[0,1]$, and the $(f \lambda, \lambda)$-distribution $\mu$ has $(\tau, 1)$-moments on $\mathrm{C}_{s}[0,1]$. The $(f \lambda, \lambda)$-distribution $\mu$ on $R^{[0,1]}$ is $\sigma$-additive, but it is not cylindrically concentrated on bounded sets in $R^{[0,1]}$.

Let $\zeta \in \mathrm{C}_{s}[0,1]^{\prime}$ be the evaluation functional at 0 ; that is, $\langle g, \zeta\rangle=g(0)$ for all $g \in \mathrm{C}_{s}[0,1]$. Then

$$
\nu=1 / 2 \zeta \mu
$$

is a cylindrical probability on $\mathrm{C}_{s}[0,1]$ such that

$$
\nu(|\zeta|)=1 / 2 \mu\left(\zeta^{2}\right)=\infty .
$$

Thus $\nu$ fails to have first order moments, but by suitably "smoothing" the function $f:[0,1] \rightarrow R^{[0,1]}$ at 0 , we can find a sequence $\nu_{n}, n=1,2, \cdots$ of cylindrical probabilities with $(\tau, 1)$-moments on $\mathrm{C}_{s}[0,1]$ such that $\nu_{n} \rightarrow \nu$ 
in the topology $\sigma_{c}$ on $\mathrm{P}\left(\mathrm{C}_{s}[0,1]\right)$.

Now each $\nu_{n}$ will not be $\sigma$-additive on $\mathrm{C}_{s}[0,1]$, but by mapping $\mathrm{C}[0,1]$ into $\mathrm{L}^{1}(\lambda)$ via the canonical inclusion $u$, each $\nu_{n} \circ u^{-1}$ extends to a Radon measure on $L^{1}(\lambda)$, as does $\nu \circ u^{-1}$. In this case, we can verify this directly, but $u$ is absolutely summing so Theorem 5.5 also covers the situation.

We now consider the case when the underlying locally convex space $E$ has a Radon-Nikodým property; that is, all vector measures in a certain class are differentiable with respect to scalar measures. These notions seem to be tied to an underlying boundedness structure in the locally convex space.

Definition 5.7. Let $\mathscr{B}$ be a complete convex bornology in the locally convex space $E$. Then $\mathscr{B}$ is said to have the Radon-Nikodym property if for every measurable space $(\Omega, \mathscr{S})$, and every vector measure $m: \mathscr{S} \rightarrow E$ absolutely continuous with respect to a scalar measure $\mu: \mathscr{S} \rightarrow[0,1]$ such that $m$ has finite $B$-variation, $B \in \mathscr{B}$ [7], there exists a function $f: \Omega \rightarrow E$ with the following properties:

(i) $m=f \mu$;

(ii) there exists a partition $\Omega_{n} \in \mathscr{S}, n=1,2, \ldots$ of $\Omega$ and closed disked sets $B_{n} \in \mathscr{B}, n=1,2, \cdots$ containing $B$ such that $f: \Omega_{m} \rightarrow E_{B_{n}}$ is Bochner $\mu$-integrable in $E_{B_{n}}$ [3].

We say that $\mathscr{B}$ as the strict Radon-Nikodým property if for each closed, disked set $B \in \mathscr{B}$, there exists a single closed, disked set $C \in \mathscr{B}$ containing $B$ such that vector measures with finite $B$-variation have a Bochner integrable density in $E_{C}$.

The Radon-Nikodým property for the bornology $\mathscr{B}_{0}$ of all bounded subsets of a Banach space is systematically treated in the monograph of Diestel and Uhl [3]. Obviously the Radon-Nikodým property and strict Radon-Nikodým property coincide for this case. It suffices to establish either of these properties for the case when $(\Omega, \mathscr{S})=([0,1], \mathscr{B O}[0,1])$ and is the Lebesgue measure on $[0,1]$.

We are really only concerned with the Radon-Nikodým property, but bornologies with the strict Radon-Nikodým property have been characterized in terms of geometric criteria. To see this, call a continuous linear map $u: E \rightarrow E(\mathscr{B})$ denting $[(\mathscr{B}) \sigma$-denting] if for every closed disked set $B \in \mathscr{B}$, there exists a closed disked set $C \in \mathscr{B}$ such that $u(B) \subset C$ and $u(B)$ is a dentable ( $\sigma$-dentable) subsets of $E_{C}$ (for details see [3]). 
Bornologies with the strict Radon-Nikodým property have similar properties to conuclear bornologies, except that nuclear maps are replaced by denting maps. Indeed, let us say that the complete, convex bornology $\mathscr{B}$ has the strict Lebesgue-Nikodym property if for every closed, disked $B \in \mathscr{B}$, there exists a closed disked set $C \in \mathscr{B}$ containing $B$ such that every $E_{B}$-valued vector measure has a strongly measurable [3] density in $E_{C}$. Then it follows from [14] and Theorem 4.6 that $\mathscr{B}$ is conuclear if and only if it has the strict Lebesgue-Nikodym property.

Spaces with the strict Radon-Nikodým property (for their bounded sets) therefore constitute a wider class than those for which the strict Lebesgue-Nikodým property holds. In particular, only finite dimensional Banach spaces have the Lebesgue-Nikodým property.

The relationship between the variation of vector measures and cylindrical concentration of cylindrical probabilities [7] yields the following characterization of the Radon-Nikodým property.

TheOREM 5.8. Let $\mathscr{B}$ be a complete, convex bornology in the locally convex space $E$. The following conditions are equivalent.

(i) $\mathscr{B}$ has the Radon-Nikodým property.

(ii) Each cylindrical probability $\mu \in \mathrm{P}(E)$ with $(\tau, 1)$-moments which is cylindrically concentrated on the family $\mathscr{B}$ is cylindrically concentrated on $\mathscr{B}$-strictly (weakly) compact subsets of $E$.

Cylindrical probabilities satisfying (ii) therefore extend to $\mathscr{B} \mathscr{C}$-regular Borel measures on $E_{\beta}$. The extension of this result to the limiting case has the following form. The method of proof is by now familiar and is accordingly omitted.

THEOREM 5.9. Let $\mathscr{B}$ be a complete convex bornology in the locally convex space $E$. Suppose that $\mathscr{B}$ has the Radon-Nikodým property. Assume also that $\mu \in \mathrm{P}(E)$ has one of the following properties.

(i) There exists a countable net $\left(\mu_{i}\right)_{i \in I}$ of cylindrical probabilities with $(\tau, 1)$-moments on $E$, each of which is cylindrically concentrated on the family $\mathscr{B}$, such that $\mu_{i} \rightarrow \mu$ in $\sigma_{c}$.

(ii) There exists a countable, uniformly cylindrically additive net $\left(\mu_{i}\right)_{i \in I}$ $\in \mathrm{P}(E)$ with $(\tau, 1)$-moments, each of which is cylindrically concentrated on $\mathscr{B}$, such that $\mu_{i} \rightarrow \mu$ in the cylindrical topology $\gamma$.

Then $\mu$ extends to a $\mathscr{B} \mathscr{C}$-regular Borel measure on $E_{\beta}$.

The converse clearly holds by limiting the extension of $\mu$ to compact 
sets of positive measure

COROLLARY 5.10. Let $\mathscr{B}$ be a complete, convex bornology with the Radon-Nikodým property in $E$.

If $\mu \in \mathrm{P}(E)$ is cylindrically concentrated on $\mathscr{B}$ and scalarly concentrated on weakly compact convex subsets in $E$, then $\mu$ extends to a $\mathscr{B} \mathscr{C}$-regular Borel measure on $E_{\beta}$.

Proof. Let $\nu$ denote the extension of the cylindrical probability induced by $\mu$ on $E_{\sigma}^{\prime \prime}$ to a Radon measure on $E_{\sigma}^{\prime \prime}$. There exists weakly compact subsets $\bar{B}_{n}$ of $E_{\sigma}^{\prime \prime}, n=1,2, \cdots$ which are the closures of the disked sets $B_{n} \in \mathscr{B}$ in $E_{o}^{\prime \prime}$ such that

$$
\nu\left(\bar{B}_{n}\right) \geqq 1-1 / 2 n, \quad n=1,2, \cdots .
$$

Let $\nu_{n}=\chi_{\bar{B}_{n}} \nu / \nu\left(\bar{B}_{n}\right), n=1,2, \cdots$ and put

$$
\mu_{n}(A \cap E)=\nu_{n}(A), \quad A \in \mathscr{Z}\left(E_{\sigma}^{\prime \prime}\right), \quad n=1,2, \cdots .
$$

If $j_{n}: L^{\circ}(\nu) \rightarrow L^{\circ}\left(\nu_{n}\right)$ is the restriction map, and if $M: E^{\prime} \rightarrow L^{\circ}(\nu)$ is the random linear function associated with $\mu$, then $M_{n}=j_{n} \circ M$ is the random linear function associated with $\mu_{n}, n=1,2, \cdots$.

Moreover, $M_{n}\left(E^{\prime}\right) \subset L^{\infty}\left(\nu_{n}\right)$ and

$$
M_{n}: E_{\beta}^{\prime} \rightarrow L^{\infty}\left(\nu_{n}\right)
$$

is continuous. Now $M: E_{\tau}^{\prime} \rightarrow L^{\circ}(\nu)$ is continuous because $\mu$ is scalarly concentrated on weakly compact convex sets [13] p. 265, so $M_{n}: E_{\tau}^{\prime} \rightarrow L^{\circ}\left(\nu_{n}\right)$ is also continuous. It follows from Theorem 2.4 that each $\mu_{n} \in \mathrm{P}(E)$ has $(\tau, 1)$-moments and is cylindrically concentrated on $2 B_{n}$. Clearly $\mu_{n} \rightarrow \mu$ in $\sigma_{c}$ and an application of Theorem 5.9 finishes the proof.

The Banach space version of Corollary 5.10 was obtained by W. Schachermayer [9]. The bornological completeness theorem mentioned in Section 2 is used in an essential way. For probability theory on, say, a space of operators, it is crucial to assume only local completeness and not completeness of the whole space (it will only be quasicomplete in cases of interest).

Suppose that the space $F$ is quasicomplete and that the family $\mathscr{B}_{0}$ of bounded subsets of $F$ has the Radon-Nikodým property. Let $u: E \rightarrow F$ be a totally summing map from the Banach space $E$ into $F$. Now an ab- 
solutely summing map between Banach spaces is weakly compact (by virtue of the factorization through an $\mathrm{L}^{\infty}$ and $\mathrm{L}^{1}$ space), so Theorems 5.2 and 5.9 show that for any cylindrical probability $\mu \rightarrow \mathrm{P}(E)$ satisfying conditions $\mathrm{N}_{1}$ or $\mathrm{N}_{2}$ or Theorem 4.1, for countable nets, $\mu \circ u^{-1}$ extends to a $\mathscr{B}_{0} \mathscr{C}$-regular Borel measure on $F$. Indeed, it follows from [12] that this is a characterization of quasicomplete spaces $F$ for which $\mathscr{B}_{0}$ has the Radon-Nikodým property.

The arguments used here concerning vector measures are very general, but they are of relevance to most spaces which arise in applications of functional analysis. For example, it is well-known that each of the common spaces of distributions is nuclear, and the collection of bounded sets in these spaces is conuclear.

In quantum field theory, one is forced to deal with such things as operator-valued distributions; that is, writing $\mathscr{S}$ for the space of rapidly decreasing functions, $E$ for the associated Hilbert space of states, analysis on the locally convex space $\mathscr{L}_{s}\left(\mathscr{S}, \mathscr{L}_{s}(E)\right)$ is relevant [5]. For example, if the statistical behaviour of a large collection of relativistic quantum fields were to be determined, then a probability measure needs to be defined on the space $\mathscr{L}_{s}\left(\mathscr{S}, \mathscr{L}_{s}(E)\right.$ ) (or some subspace of it) in the same manner in which classical statistical mechanics deals with the collection of continuous evolving configurations of a dynamical system. The mathematical foundations of such a quantum statistical field theory are not yet at hand, but the techniques developed here for establishing the regularity of cylindrical probabilities on vector spaces may well be relevant in future.

This work is a portion of the author's doctoral dissertation at The Flinders University of South Australia under the supervision of Igor Kluvánek. The guidance of Professor Kluvánek is gratefully acknowledged.

\section{References}

[1] A. Badrikian, Seminaire sur les fonctions eleatoires et les mesures cylindriques, Lecture Notes in Math., 139 (1970).

[2] G. Y. H. Chi, On the Radon-Nikodým theorem in locally convex spaces, Lecture Notes in Math., 541 (1975), 199-210.

[3] J. Diestel and J. J. Uhl Jr., Vector Measures, Mathematical Surveys No. 15, American Math. Soc., Providence, 1977.

[4] N. Dunford and J. R. Schwartz, Linear Operators, Pt I, Interscience, New York, 1958.

[5] J. Glimm and A. Jaffe, Quantum Physics: A Functional Integral Point of View, Springer-Verlag, New York, 1981. 
[6] B. Jefferies, Collectively measurable sets and abstract Wiener spaces, Indiana Univ. Math. J. 33 (1984), 143-158.

[7] - The variation of vector measures and cylindrical concentration, Illinois $J$. Math., 30 (1986), 511-526.

[8] A. Pietsch, Nuclear Locally Convex Spaces, Springer-Verlag, New York, 1972.

[9] W. Schachermayer, Mesures cylindriques sur les espaces de Banach qui ont le Radon-Nikodým, C.R. Acad. Sci. Paris, Ser. A282 (1976), 227-229.

[10] H. H. Schaefer, Topological Vector Spaces, Springer-Verlag, New York, 1971.

[11] L. Schwartz, Seminaire Laurent Schwartz, Ecole Polytechnique, exposes no 3, 4, $11,12,13$, annee 1969-1970.

[12] - Seminaire Maurey-Schwartz, Ecole Polytechnique, exposes no 4, 5, 6, annee 1974-1975.

[13] — Radon Measures in Arbitrary Topological Spaces, Tata Inst. of Fund. Res., Oxford Univ. Press, 1973.

[14] E. Thomas, The Lebesgue-Nikodým Theorem for Vector-Valued Radon Measures, Mem. Amer. Math. Soc., no 139 (1974).

[15] N. N. Vakhaniya, V. I. Tarieladze and A. Tortrat, On the continuity of random linear functionals, Theory Prob. Appl., 26 (1981), 167-173.

Department of Mathematics

University of Wollongong

P. O. Box 1144, Wollongong

N.S.W. 2500

Australia 